\title{
Cell Diversity and Connection Specificity between Callosal Projection Neurons in the Frontal Cortex
}

\author{
Takeshi Otsuka and Yasuo Kawaguchi \\ Division of Cerebral Circuitry, National Institute for Physiological Sciences, Department of Physiological Sciences, Graduate University for Advanced \\ Studies (SOKENDAI), Okazaki, Aichi 444-8787, Japan, and Japan Science and Technology Agency, Core Research for Evolutional Science and Technology, \\ Tokyo 102-007, Japan
}

Recent advances have established that intralaminar and interlaminar excitatory networks between neocortical pyramidal cells are specialized into subnetworks. Here, we have investigated how the commissural system organizes the intracortical excitatory subnetworks to communicate between cortical hemispheres. Whole-cell recordings were obtained from callosal projection neurons [commissural (COM) cells], identified by in vivo injection of retrograde fluorescent tracer into one hemisphere, in rat frontal cortical slices. We found that layer V (L5) COM cells were heterogeneous in physiological and morphological properties that correlated with projection patterns to contralateral and ipsilateral cortical areas. The probability of synaptically connected pairs of L5 COM cells was higher in cell pairs of the same firing subtypes than that in different cell subtype pairs. In interlaminar connections, layer II/III (L2/3) COM cells preferentially innervated L5 COM cells. Moreover, pairs of the same L5 COM subtypes were more likely to share inputs from L2/3 COM cells than were different COM subtype cell pairs. In addition, common inputs from L2/3 COM cells were frequently observed in L5 pairs of corticopontine cells and given firing subtypes of COM cells. Our results suggest that callosal communications are achieved via several distinct COM cell subnetworks differentiated according to the ipsilateral corticocortical and subcortical projection patterns.

\section{Introduction}

The neocortex is a layered structure containing vertical columnar assemblies of numerous types of excitatory and inhibitory neurons (DeFelipe and Fariñas, 1992; Kawaguchi and Kubota, 1997; Mountcastle, 1997). As expected from columnar organization, cortical cells are well connected vertically in a direction-selective manner (Weiler et al., 2008; Lefort et al., 2009). Inputs from the thalamus to neurons in superficial cortical layers are relayed to neurons in deeper layers that then provide output to various subcortical areas (Bureau et al., 2006; Lübke and Feldmeyer, 2007). How do intracortical circuits process the information for diverse outputs to multiple projection areas? Recent studies have shown that local circuits of pyramidal cells within the column are clustered into subnetworks in intralaminar and interlaminar connections. In intralaminar connections, pairs of connected layer V (L5) pyramidal cells are more likely to form connections onto a third cell than are unconnected cell pairs (Song et al., 2005). Furthermore, intralaminar connections between L5 pyramidal cells were made, depending on their subcortical targets (Morishima and Kawaguchi, 2006; Brown and Hestrin, 2009). In interlaminar connections, probabilities of divergent and conver-

Received Nov. 3, 2010; revised Jan. 6, 2011; accepted Jan. 14, 2011.

This work was supported by Japan Science and Technology Agency, Core Research for Evolutional Science and Technology; Grant-in-Aids for Scientific Research from the Ministry of Education, Culture, Sports, Science, and Technology and the Research Program of Hayama Center for Advanced Studies of Sokendai. We thank Allan Gulledge for helpful comments on the manuscript.

Correspondence should be addressed to Yasuo Kawaguchi, Division of Cerebral Circuitry, National Institute for Physiological Sciences, 5-1 Myodaiji-Higashiyama, Okazaki, Aichi 444-8787, Japan. E-mail: yasuo@nips.ac.jp.

DOI:10.1523/JNEUROSCI.5795-10.2011

Copyright $\odot 2011$ the authors $\quad 0270-6474 / 11 / 313862-09 \$ 15.00 / 0$ gent inputs to pyramidal cells depend on the connectivity patterns of recipient and projecting cell pairs (Yoshimura et al., 2005; Kampa et al., 2006; Otsuka and Kawaguchi, 2008). Connection patterns from layer II/III (L2/3) to L5 pyramidal cells are specified by L5 pyramidal subtypes and their sublayer positions (Otsuka and Kawaguchi, 2008; Anderson et al., 2010). Moreover, interlaminar connections from L2/3 to L5 pyramidal cells form subnetworks, depending on L5 pyramidal subtypes that correlate with subcortical projection targets (Otsuka and Kawaguchi, 2008). These results suggest that intralaminar and interlaminar excitatory networks are segregated into functional channels corresponding to subcortical targets.

The cortex is composed of two hemispheres and communicates the information between them via the corpus callosum. Several studies have suggested that interhemispheric cortical communications participate in motor coordination (Bury and Jones, 2002) as well as higher associative and cognitive function (Hasegawa et al., 1998; Larson et al., 2002). Dysfunction of information transfer between cortical hemispheres by callosal transaction causes dramatic deficits in sensation and perception (Gazzaniga, 2005). These observations indicate the importance of interhemispheric cortical communications. Several studies have shown that callosal fibers innervate both pyramidal cells and inhibitory interneurons (Kawaguchi, 1992; Carr and Sesack, 1998; Karayannis et al., 2007; Petreanu et al., 2007). However, it remains unknown how the commissural system organizes the intracortical excitatory subnetworks. In this study, we investigated physiological and morphological properties of callosal projection neurons [commissural (COM) cells] and their synaptic connection patterns. We found that L5 COM cells are heteroge- 
neous in physiological and morphological properties that correlated with projection patterns to contralateral and ipsilateral cortical areas. Intralaminar and interlaminar connections between COM cells depended on L5 COM subtypes. Moreover, specific L5 COM subtypes formed interlaminar subnetworks with L5 pyramidal cells projecting to the ipsilateral subcortical area. Our results suggest that L5 COM subtypes make several communication channels, partially segregated according to their projection patterns, between cortical hemispheres.

\section{Materials and Methods}

In vivo retrograde labeling and whole-cell recordings in slice. All experiments were conducted in compliance with the guidelines for The Institutional Animal Care and Use Committee of National Institutes of Natural Sciences. To identify pyramidal cells projecting to a particular brain area, retrograde fluorescent tracers were injected in vivo into the object brain area of Wistar rats anesthetized with ketamine $(40 \mathrm{mg} / \mathrm{kg}$, i.m.) and xylazine ( $4 \mathrm{mg} / \mathrm{kg}$, i.m.). To identify callosal projection pyramidal cells (COM cells), Alexa Fluor 555-conjugated cholera toxin subunit B (CTB) (Invitrogen) or rhodamine-labeled latex microspheres (RLMs) (Lumafluor) was injected into the contralateral frontal cortex (4 $\mathrm{mm}$ anterior to bregma, $1.5-2.5 \mathrm{~mm}$ lateral to bregma, and $0.5-0.8 \mathrm{~mm}$ depth) from the recording site with air-pressure applications using glass pipettes. Alexa Fluor 555-conjugated CTB and RLMs were used when COM cells and pyramidal cells projecting to the ipsilateral pontine nuclei (5.6 $\mathrm{mm}$ posterior to bregma, $0.5-1 \mathrm{~mm}$ lateral to bregma, and $9 \mathrm{~mm}$ depth) were simultaneously identified in the same preparation. Cells labeled by CTB and RLMs were clearly distinguished from each other in the same preparation (Morishima and Kawaguchi, 2006). In the case of double-labeling slice experiments, Alexa Fluor 488- or FITC-conjugated CTB was injected into ipsilateral primary somatosensory (S1) $(0.5 \mathrm{~mm}$ posterior to bregma, 3-5 $\mathrm{mm}$ lateral to bregma, and $0.5-0.8 \mathrm{~mm}$ depth) or posterior parietal cortex (PPC) $(4 \mathrm{~mm}$ posterior to bregma, $3 \mathrm{~mm}$ lateral to bregma, and $0.5-0.8 \mathrm{~mm}$ depth), or ipsilateral striatum (ipsiStr) ( $0.8 \mathrm{~mm}$ posterior to bregma, $2.5 \mathrm{~mm}$ lateral to bregma, and $4 \mathrm{~mm}$ depth) from the recording site, while red fluorescent tracer was injected into the contralateral frontal cortex. In the case of striatum injection, the cortex was removed by suction. For injections into the frontal cortex, glass pipettes containing the tracer were angled at $20-25^{\circ}$ to the rostral direction from the vertical. After tracer injection, animals were fed for $2-3 \mathrm{~d}$ as a recovery and tracer transportation period before slice experiments.

Brain slices containing the frontal cortex were obtained from male and female animals aged postnatal 19-23 d, as described previously (Otsuka and Kawaguchi, 2008, 2009). After cutting the brain, slices (300 $\mu \mathrm{m}$ thick) were incubated for at least $1 \mathrm{~h}$ in oxygenated artificial CSF (ACSF) composed of the following (in mM): $126 \mathrm{NaCl}, 2.5 \mathrm{KCl}, 1.25 \mathrm{NaH}_{2} \mathrm{PO}_{4}, 1$ $\mathrm{MgCl}_{2}, 2 \mathrm{CaCl}_{2}, 26 \mathrm{NaHCO}_{3}$, and 10 glucose, $\mathrm{pH} 7.4(310 \pm 5 \mathrm{mOsm} / \mathrm{L}$; bubbled with $\left.95 \% \mathrm{O}_{2} / 5 \% \mathrm{CO}_{2}\right)$. Whole-cell recordings were obtained from retrogradely labeled cells, identified under epifluorescent illumination $[40 \times$ objective lens, numerical aperture (NA) $=0.8$; Axioskop, Zeiss], with the recording pipettes filled with a solution containing the following (in mM): 130 potassium methylsulfate, $0.5 \mathrm{EGTA}, 2 \mathrm{MgCl}_{2}, 2$ $\mathrm{Na}_{2} \mathrm{ATP}, 0.2$ GTP, 20 HEPES, 0.1 leupeptin and $0.75 \%$ biocytin, $\mathrm{pH} 7.2$ $(290 \pm 5 \mathrm{mOsm} / \mathrm{L})$. Cells labeled by green fluorescent tracers were observed under a $40 \times$ objective lens with larger numerical aperture $(\mathrm{NA}=$ 1.0, Zeiss). To examine interlaminar connections from L2/3 to L5 cells, we focally applied glutamate to L2/3 cells, as described previously (Otsuka and Kawaguchi, 2008, 2009). Sodium glutamate (1 mM) was dissolved in the ACSF and filled into the same pipettes as those for wholecell recordings. To evoke a spike in a selected cell, the pipettes filled with glutamate were positioned within $10 \mu \mathrm{m}$ from the cell soma. Glutamate was ejected using the air-puffer ( $50 \mathrm{~ms}$ duration and pressure $<10 \mathrm{psi}$ ). Data are represented as mean $\pm S D$, and statistical differences between samples were tested using ANOVA with Tukey post hoc tests, unless otherwise mentioned. Significance was accepted when $p<0.05$.

Morphological analysis. Slices containing cells intracellularly labeled with biocytin were fixed with a solution containing $4 \%$ paraformalde- hyde, $1.25 \%$ glutaraldehyde, and $0.2 \%$ picric acid in phosphate buffer (PB) and resectioned at a thickness of $50 \mu \mathrm{m}$. Neurons labeled with biocytin were visualized by the avidin-biotin-horseradish peroxidase reaction. After staining, sections were postfixed in $1 \% \mathrm{OsO}_{4}$ in $\mathrm{PB}$ containing 7\% glucose and coverslipped with Epon after dehydration.

The Neurolucida system (MicroBrightField) was used for a reconstruction of the stained cell. Stained cells were observed with a $60 \times$ objective lens followed by $1.25 \times$ magnification. Apical and basal dendrites of reconstructed cells were analyzed using Neurolucida Explorer (MicroBrightField). Internode intervals were defined as the length between two successive nodes (branch points) along the dendrite, including those from the soma origin to the first node. Internode tortuosity was measured as the ratio of the length of internode interval divided by the direct distance between nodes. The basal field was the area of the plane perpendicular to the pia-white matter axis onto which the basal dendrites were projected.

\section{Results}

Pyramidal cells projecting to the other hemisphere through corpus callosum (COM cells) were identified by the perpendicular injection of retrograde fluorescent tracer into the frontal cortex (Fig. 1A). Two or three days after in vivo tracer injection, retrogradely labeled cells were found in both L2/3 and L5 of the contralateral frontal cortex and distributed vertically in a columnar manner (Fig. $1 B$ ).

\section{Physiological and morphological properties of L5 COM cells} We have previously described that L5 pyramidal cells in the rat frontal cortex can be classified into three subtypes based on their firing properties (Otsuka and Kawaguchi, 2008). Whereas the firing patterns of L5 pyramidal cells projecting to the ipsilateral pons or the contralateral striatum correlate with their axonal targets, L5 COM cells exhibited heterogeneous firing patterns, including three subtypes defined by the firing patterns in the response to the current pulse injections (Otsuka and Kawaguchi, 2008). One class of L5 COM cells gradually reduced spike frequencies during current pulse injection [fast spike frequency adaptation (FA) type] (Fig. 2A, lower trace). The other classes of L5 COM cells showed repetitive spike discharges with relatively constant spike frequencies during depolarization [slow spike frequency adaptation (SA) type] (Fig. 2A, upper trace). Some of them showed initial burst (doublet) spikes at the beginning of the spike train [slow spike frequency adaptation with initial doublet spikes (SA-d) type] (Fig. $2 A$, middle trace).

To quantify firing subtypes of L5 COM cells, we obtained recordings from retrogradely labeled cells and calculated spike frequencies ( $f$ ) from interspike intervals in response to the current pulse injection (amplitude, $500 \mathrm{pA}$; duration, $1 \mathrm{~s}$ ) in individual cells (Fig. $2 B$, inset). From firing frequencies calculated from the first, second, and seventh interspike intervals ( $\mathrm{f} 1, \mathrm{f} 2, \mathrm{f} 7)$, we obtained the adaptation index (f7/f2) and the burst index (f1/f2). Shown in Figure $2 B$ is the relationship between the adaptation index and $\mathrm{fl}$ in individual cells. Three firing types were quantitatively distinguishable by these parameters, in a similar way to our previous study (Otsuka and Kawaguchi, 2008). The adaptation index of FA type L5 COM cells was lower than that of other types (L5 COM cells: SA, $0.82 \pm 0.12$; SA-d, $0.82 \pm 0.14$; FA, $0.18 \pm$ $0.16 ; p<0.001)$. The burst index of SA-d type L5 COM cells was significantly higher than that in other types (L5 COM cells: SA, $1.5 \pm 0.24 ;$ SA-d, $5.47 \pm 2.42 ; \mathrm{FA}, 1.63 \pm 0.46 ; p<0.001)$. The proportion of SA, SA-d, and FA subtypes in recorded L5 COM cells were $32,14.9$, and $53.1 \%(n=138,64$, and 229 , respectively) (Fig. 2D). These results suggest that L5 COM cells consist of three firing subtypes. 
It is known that COM cells send axon collaterals to ipsilateral other cortical areas (Veinante and Deschênes, 2003; Mitchell and Macklis, 2005). To characterize the relationship between projection areas and firing subtypes of L5 COM cells, two different retrograde fluorescent tracers were injected into the contralateral frontal cortex and ipsilateral S1 or PPC from the recording cortical hemisphere. We then obtained recordings from double-labeled L5 cells that project to both cortical hemispheres (Fig. 2C). In both cases where L5 cells project to the contralateral frontal cortex and ipsilateral S1 or PPC, almost all recorded cells showed FA type firing patterns (30 of 33 $\mathrm{COM} / \mathrm{S} 1$ cells and 29 of $31 \mathrm{COM} / \mathrm{PPC}$ cells) (Fig. $2 D$ ). The rest were of SA type firing patterns, and no SA-d type cells were found in either case. We also obtained recordings from L5 pyramidal cells that project to the contralateral frontal cortex and ipsi-Str (COM/ipsi-Str), identified by tracer injections. In contrast to $\mathrm{COM} / \mathrm{S} 1$ and COM/PPC cases, COM/ ipsi-Str cells contained three firing subtypes (Fig. 2D). The proportion of subtypes in recorded L5 COM/ipsi-Str cells were similar to that in L5 COM cells, and showed 23.5 for SA, 14.7 for SA-d, and $61.8 \%$ for FA types $(n=8,5$, and 21 , respectively). These results suggest that SA and SA-d type L5 COM cells project to the contralateral cortical hemisphere without axon collaterals to S1/PPC.

We next analyzed the morphological properties of L5 COM cells. Dendritic arborizations of L5 COM cells were different between firing subtypes. The apical tuft length of SA and SA-d type L5 COM cells was longer than that of FA type cells (Table 1). The total length of apical tufts in layer I gradually decreased according to the somatic depth in all three types (Fig. $3 B, C$ ). No correlation was found in the relationship between the adaptation index (f7/f2) and the tuft length in FA type COM cells. The apical tufts of SA and SA-d type COM cells originated from the main apical shafts at a greater depth than those of FA type cells (Fig. $3 A, B)$. In contrast, morphologies of the basal dendrites were similar among L5 COM subtypes (Table 1). It has been shown that a subset of COM cells projects to the contralateral striatum (Wilson, 1987). L5 pyramidal cells that project to the contralateral striatum [crossed corticostriatal (CCS) cells] had slender type apical dendrites and majority of them showed FA type firing properties (Morishima and Kawaguchi,
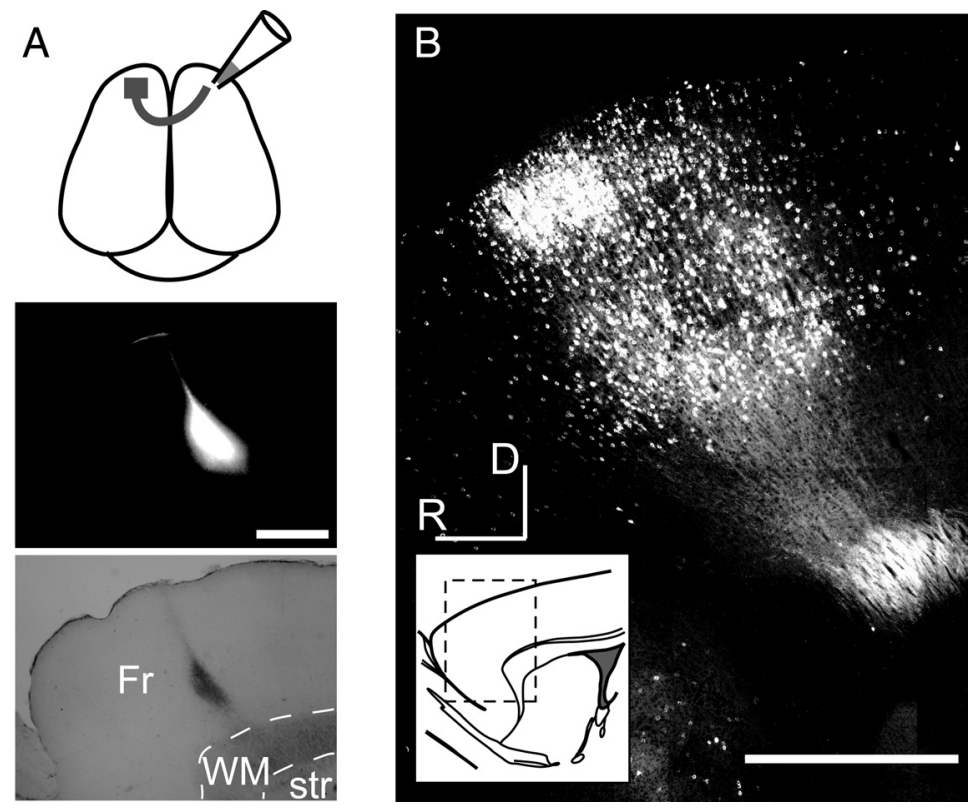

Figure 1. Retrograde labeling of callosal projection neurons in the frontal cortex. $\boldsymbol{A}$, Retrograde fluorescent tracer was injected into the frontal cortex. Middle, injection site; lower, bright-field image. Fr, Frontal cortex; WM, white matter. B, Cells labeled with retrograde fluorescent tracer were distributed both in L2/3 and L5. R, Rostral; D, dorsal. Inset, Illustration of sagittal section of the brain. The box indicates the location of the image. Scale bars, $1 \mathrm{~mm}$.
A
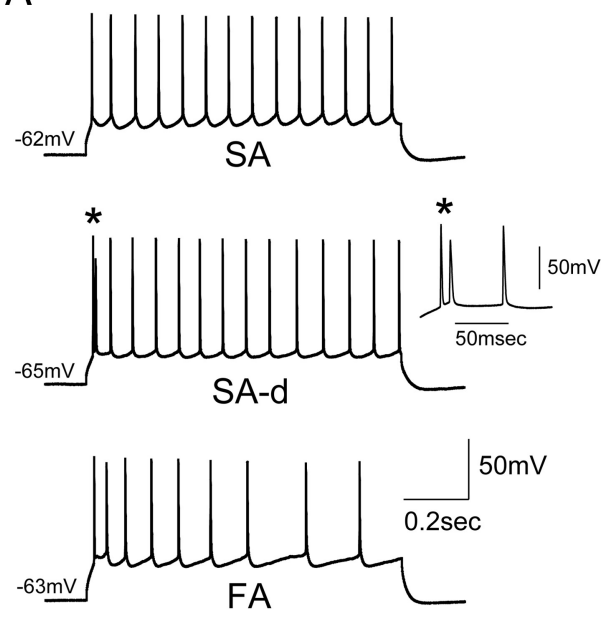

C

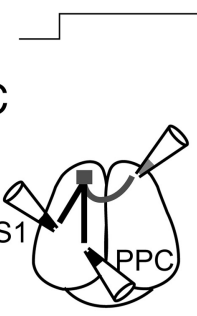

B

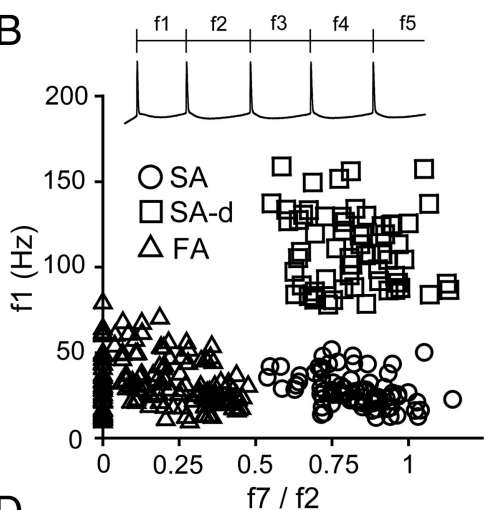

D

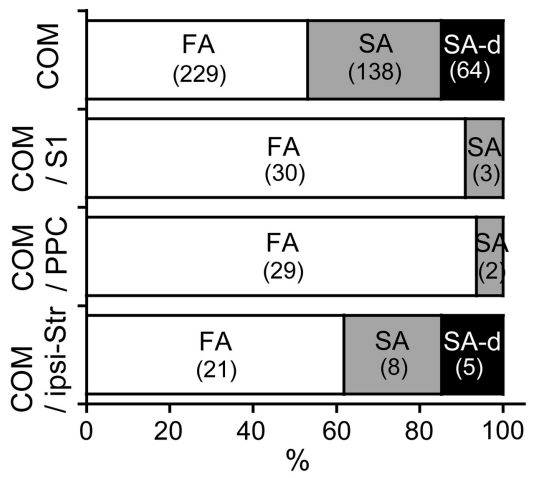

Figure 2. Three firing subtypes in $\mathrm{L} 5$ COM cells. $A$, Firing patterns of $L 5$ COM cells in response to current pulse injection ( $500 \mathrm{pA}$, $1 \mathrm{~s})$. Inset in SA-d, initial doublet $\left(^{*}\right)$ at an expanded time scale. $\boldsymbol{B}$, Relationship between adaptation index and first spike frequency (f1) during current pulse injection (500 pA, $1 \mathrm{~s})(n=85,64$, and 155 cells, for SA, SA-d, and FA type). C, Fluorescent retrograde tracers were injected to the contralateral frontal cortex and ipsilateral $S 1$ or PPC to identify $L 5$ cells projecting to both frontal cortex and S1 (COM/S1) or PPC (COM/PPC). Images were taken in a living slice. Left, DIC image. Middle, Labeling by rhodamine-labeled latex microspheres (RLMs, frontal cortex injection). Right, Labeling by cholera toxin subunit B-conjugated Alexa Fluor 488 (CTB488, S1 injection). Scale bar, $20 \mu \mathrm{m}$. D, Percentage of firing subtypes in L5 COM, COM/S1, COM/PPC, and COM cells projecting to the ipsilateral striatum (COM/ipsi-Str). 


\begin{tabular}{|c|c|c|c|c|}
\hline & \multicolumn{3}{|l|}{ Layer V } & \multirow[b]{2}{*}{ (d) Layer II/III $(n=10)$} \\
\hline & (a) $S A(n=11)$ & (b) SA-d $(n=12)$ & (c) FA $(n=18)$ & \\
\hline \multicolumn{5}{|l|}{ Somata } \\
\hline Cross-sectional area $\left(\mu \mathrm{m}^{2}\right)$ & $212.3 \pm 37.1$ & $203.9 \pm 27.1$ & $194.8 \pm 36.3$ & $129.1 \pm 24.0(d<a, b, c)^{* * *}$ \\
\hline \multicolumn{5}{|l|}{ Basal dendrites } \\
\hline Primary dendrite number & $7.1 \pm 1.3$ & $7.0 \pm 1.5$ & $7.3 \pm 1.5$ & $5.8 \pm 1.3(d<a, b, c)^{* *}$ \\
\hline Internode interval & $20.9 \pm 3.6$ & $24.2 \pm 6.1$ & $24.1 \pm 7.8$ & $21.0 \pm 5.7$ \\
\hline Internode tortuosity & $1.12 \pm 0.02$ & $1.14 \pm 0.02$ & $1.14 \pm 0.03$ & $1.13 \pm 0.02$ \\
\hline Area $\left(\times 100 \mu \mathrm{m}^{2}\right)$ & $603.1 \pm 205.6$ & $631.4 \pm 203.0$ & $688.0 \pm 188.7$ & $449.9 \pm 160.0(d<b)^{*} ;(d<c)^{* *}$ \\
\hline \multicolumn{5}{|l|}{ Apical dendrites } \\
\hline Tuft length in layer I ( $\mu \mathrm{m})$ & $1108 \pm 621$ & $1354 \pm 923$ & $612 \pm 543$ & $1689 \pm 894(c<b)^{*} ;(c<d)^{* *}$ \\
\hline Tuft origin ( $\mu \mathrm{m})$ & $-117.4 \pm 63.2$ & $-150.9 \pm 121.7$ & $-37.5 \pm 43.4$ & $-30.2 \pm 17.0(c, d<a)^{*} ;(c, d<b)^{* *}$ \\
\hline Oblique branch density $(/ 100 \mu \mathrm{m})$ & $2.6 \pm 0.8$ & $3.0 \pm 0.9$ & $2.4 \pm 0.4$ & $3.3 \pm 1.1(c<d)^{*}$ \\
\hline
\end{tabular}

Data are means \pm SD. $n$, Number of reconstructed cells. ${ }^{*} p<0.05 ;{ }^{* *} p<0.01 ;{ }^{* * *} p<0.001$ (Tukey test).

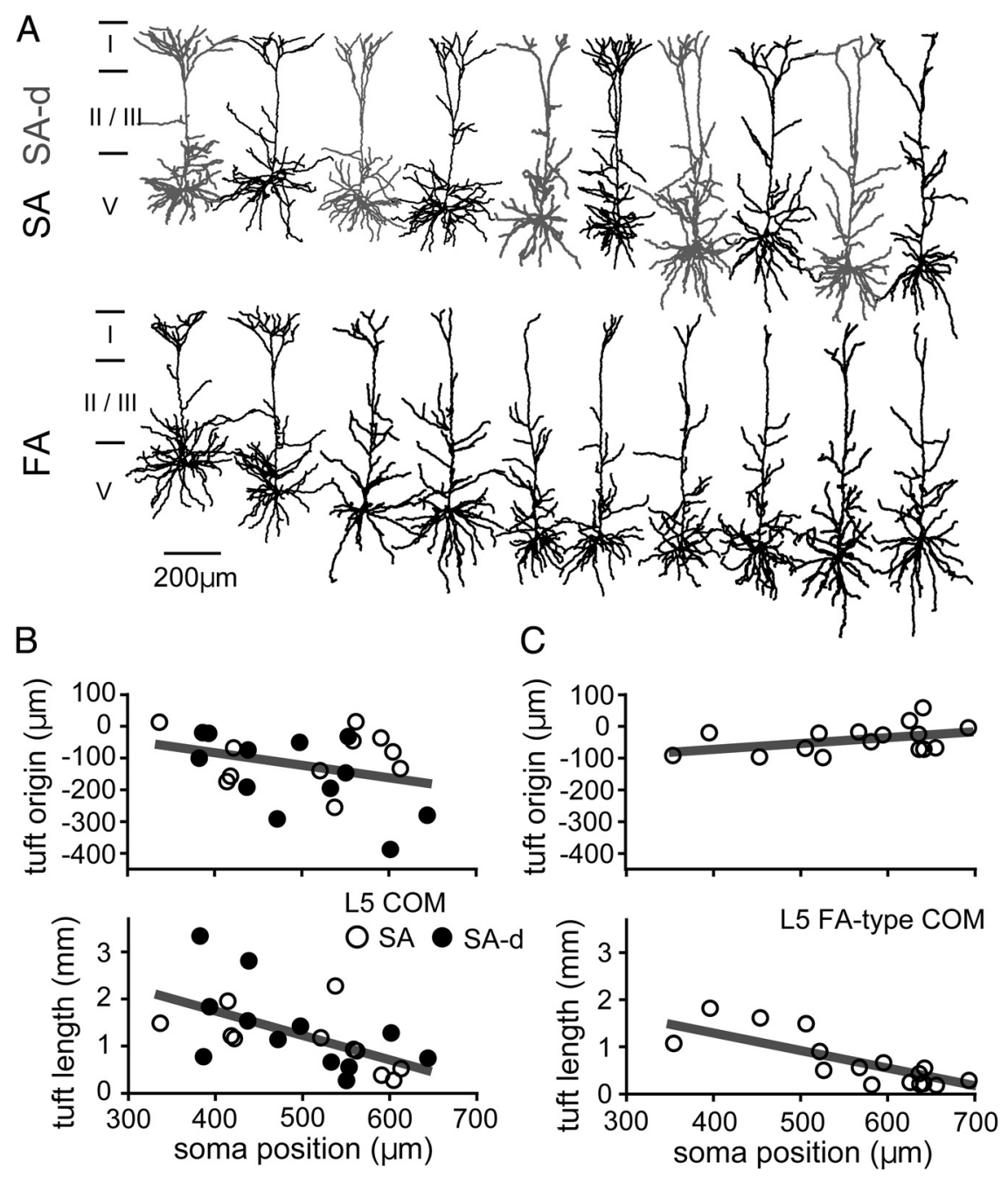

Figure 3. Morphological properties of $L 5$ COM cells. $A$, Neurolucida drawings of $L 5 S A$ and SA-d (top), and FA type (bottom) COM cells were lined up from superficial to deeper somata. Black and gray cells in the upper line are SA and SA-d type COM cells, respectively. $B, C$, Relationship between soma position and tuft origin or total length of apical tufts in layer I. Soma position was vertically measured from the border between layer I and II. $N=11,12$, and 18 cells, respectively, for SA, SA-d, and FA types.

2006; Otsuka and Kawaguchi, 2008), suggesting that CCS cells and FA type L5 COM cells overlap each other.

Physiological and morphological properties of L2/3 COM cells

We next characterized physiological and morphological properties of COM cells in L2/3. In contrast to L5 COM cells consisting of three firing subtypes, L2/3 COM cells were homogeneous in firing responses to current pulse injections (Fig. $4 A, B$ ). L2/3 $\mathrm{COM}$ cells showed repetitive spike discharge at relatively constant intervals during depolarization, corresponding to the SA firing type. Resting membrane potentials in L2/3 COM cells were lower than those in L5 COM cells $(-70.5 \pm 4.5$ for L2/3 type, $-63.7 \pm 2.6$ for L5 SA type, $-64.9 \pm 2.6$ for L5 SA-d type, and $-63.4 \pm 3.1 \mathrm{mV}$ for L5 FA type; $p<$ 0.001). All firing subtypes of L5 pyramidal cells show a prominent voltage sag, a sign of the activation of h-current (McCormick and Pape, 1990), in response to negative current pulse injections (Otsuka and Kawaguchi, 2008). However, L2/3 COM cells showed no obvious voltage sag against negative current pulse injection (Fig. $4 A$, lower traces). Voltage sag in $\mathrm{L} 2 / 3$ COM cells, measured as the voltage differences between negative peak and the steady state at the end of negative current pulse ( $-500 \mathrm{pA}$ amplitude, $1 \mathrm{~s}$ duration), was $0.74 \pm 1.37 \mathrm{mV}(n=33)$.

The soma of L2/3 COM cells was smaller than those of L5 COM cells ( $p<$ 0.001) (Table 1). Apical tufts of L2/3 COM cells originated from the main apical shafts before entering layer I and were well developed compared with those of L5 COM cells (Fig. $4 A$, Table 1 ). In contrast, the basal dendrites of L2/3 COM cells were less developed in their dendritic area, with fewer primary dendrites than those of L5 COM cells.

\section{Intralaminar connections between L5 COM cells}

Recent studies have shown that connections between L5 pyramidal cells depend on their long-range projection targets (Morishima and Kawaguchi, 2006; Brown and Hestrin, 2009). In the frontal cortex, L5 COM cells consisted of three firing subtypes that correlated with different projection patterns to the contralateral and ipsilateral cortical areas (Fig. 2). We therefore examined whether connections between L5 COM cells 
A

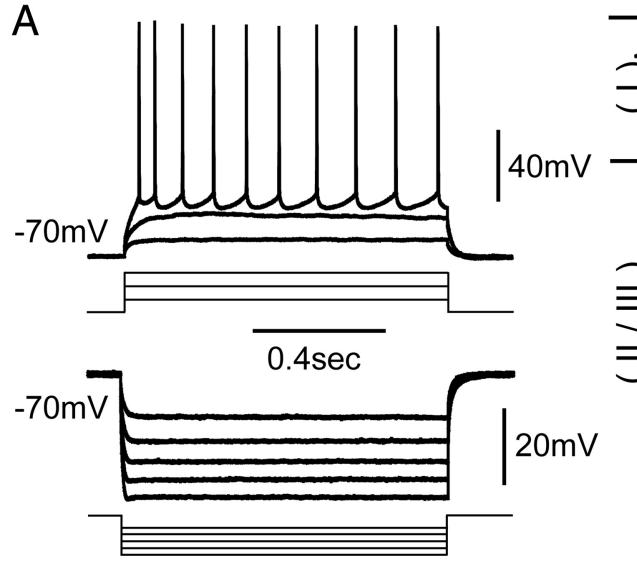

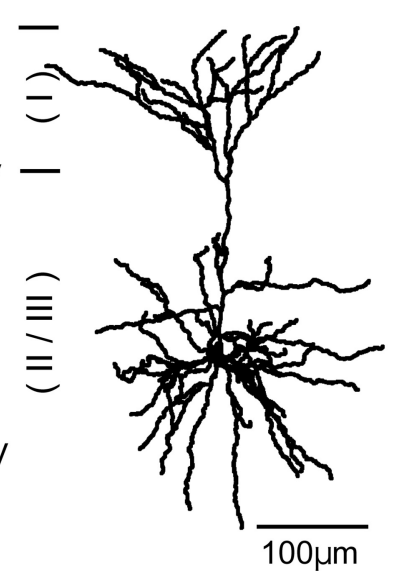

B

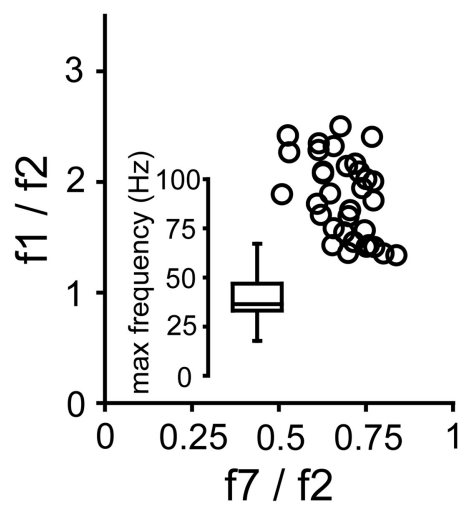

Figure 4. Firing patterns of L2/3 COM cells. $A$, Voltage traces in responses to current pulse injections in a L2/3 COM cell. Top, Positive current injections ( 100,300 , and $500 \mathrm{pA}$ amplitude, $1 \mathrm{~s}$ duration). Bottom, Negative current injections ( $-200,-300,-400,-500$, and $-600 \mathrm{pA}$ amplitude, $1 \mathrm{~s}$ duration). Right, Neurolucida drawing of a recorded L2/3 COM cell. $\boldsymbol{B}$, Relationship between burst (f1/f2) and adaptation (f7/f2) index calculated from interspike intervals discharged in response to current pulse injection ( $500 \mathrm{pA}$ amplitude, $1 \mathrm{~s}$ duration). Inset, Boxplot for maximum frequency during current pulse injection (amplitude, $500 \mathrm{pA})(33 \mathrm{~L} 2 / 3$ COM cells).

A

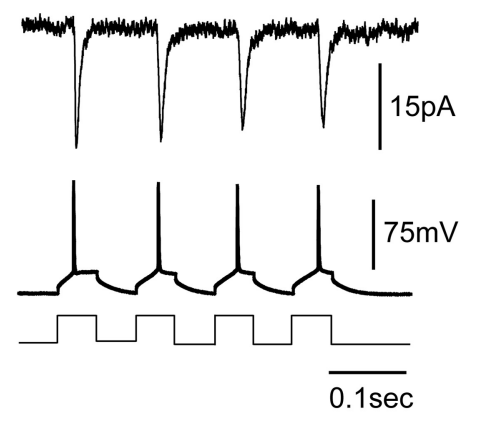

B

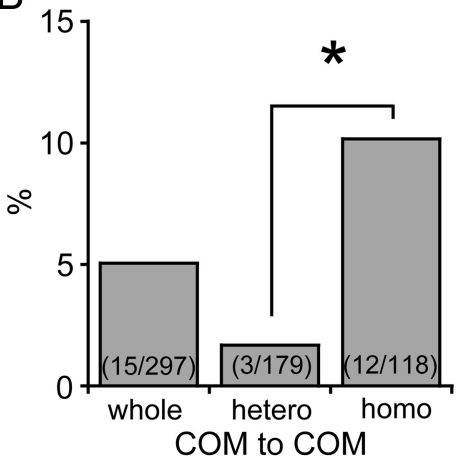

C

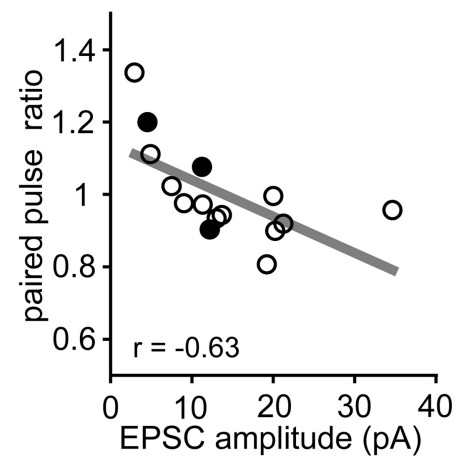

Figure 5. Intralaminar connections between $\mathrm{L} 5 \mathrm{COM}$ cells. $\boldsymbol{A}$, Dual recording from neighboring $\mathrm{L} 5$ COM cells. Spikes in the presynaptic cell (bottom trace) were elicited by current pulse injections while the postsynaptic cell was clamped at $-60 \mathrm{mV}$ (top trace). $\boldsymbol{B}$, Percentage of synaptically connected cell pairs. Whole, All cell pairs examined; hetero, cell pairs of different firing subtypes; homo, same subtype combination. ${ }^{*} p<0.05$. n1, Number of connected pairs; $\mathrm{n} 2$, number of examined pairs. $C$, Relationship between the paired-pulse ratio and the mean EPSC amplitude. Filled circles, Pairs of different subtype combinations.

are further specialized, depending on their firing subtypes. Dual whole-cell recordings were obtained from neighboring L5 COM cells. After identification of their firing subtypes, we examined synaptic connections between them (Fig. 5A). The connection probability in all pairs of L5 COM cells was 5.05\% (15 of 297 pairs) (Fig. $5 B$, whole), similar to that in the mouse visual cortex (Brown and Hestrin, 2009). However, when we divided cell pairs into two groups consisting of (1) same firing subtypes (homo pairs) and (2) different subtypes (hetero pairs), the connection probability was higher in homo pairs than that in hetero pairs $(10.17 \%$ in homo and $1.68 \%$ in hetero; $p<0.005$, Fisher exact test). Connections were found in 6 of 64 pairs in FA/FA, 4 of 32 pairs in SA/SA, and 2 of 22 pairs in SA-d/SA-d pairs. Among connection probabilities in individual subtype pairs, statistical differences against hetero pairs were observed in FA/FA and SA/SA pairs $(p<0.05$ in both cases, Fisher exact test). In hetero pairs, connections in FA/SA pairs ( 2 of 121 pairs, both from FA to SA) and SA/SA-d pairs (1 of 32 pairs, from SA to SA-d) were found, but none in FA/SA-d pairs $(n=26)$. No reciprocal connections were found in any cell pairs examined. These results suggest that connections between L5 COM cells are further specialized into subnetworks, depending on their firing subtypes.
To investigate the synaptic properties of connections between L5 COM cells, we characterized short-term plasticity for EPSCs obtained in L5 COM cell pairs. The frequency characteristics of synaptic transmission were examined at $10 \mathrm{~Hz}$ of presynaptic spike trains, while the postsynaptic cell was clamped at $-60 \mathrm{mV}$. The paired-pulse ratio, calculated as the mean amplitude of the second response divided by that of the first, depended on EPSC amplitude (Fig. 5C). Small unitary EPSCs showed paired-pulse facilitation, while large unitary EPSCs showed paired-pulse depression, similar to that in our previous study (Otsuka and Kawaguchi, 2008).

\section{Interlaminar connections from L2/3 to L5 COM cells}

We have previously shown that L2/3 pyramidal cells innervate L5 pyramidal cells with different connection probabilities, depending on L5 pyramidal firing subtypes (Otsuka and Kawaguchi, 2008). However, it remains unknown whether synaptic connections from L2/3 to L5 pyramidal cells depend on presynaptic and postsynaptic projection cell subtypes. We therefore examined whether L2/3 COM cells preferentially innervate L5 COM cells. Whole-cell recordings were obtained from L5 COM cells, while glutamate was puff applied to an $\mathrm{L} 2 / 3$ cell to trigger the spike (Fig. $6 A)$. We have previously confirmed that this technique reliably generates monosynaptic EPSCs from L2/3 to L5 cells at relatively 
A
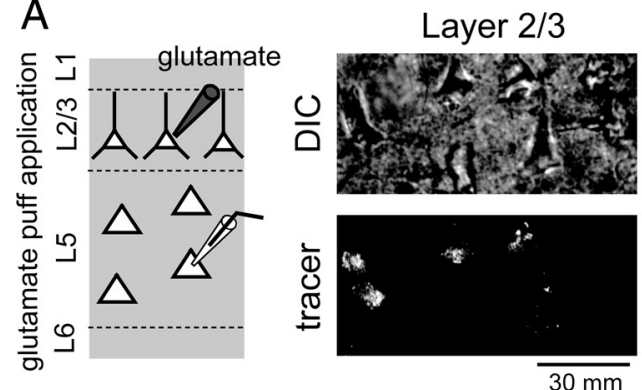

C
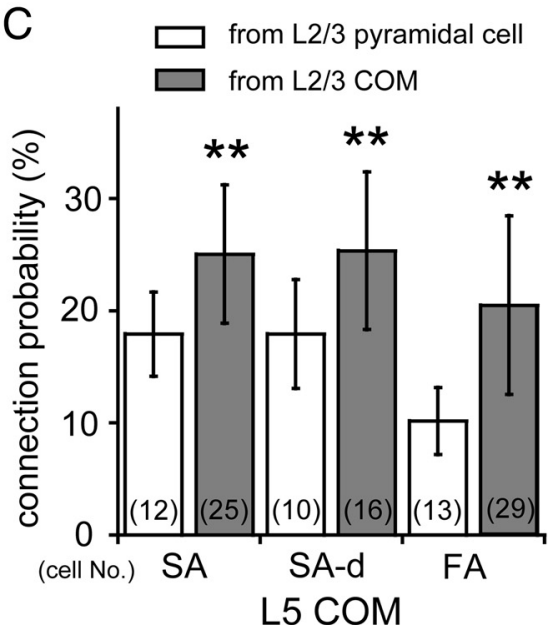

B

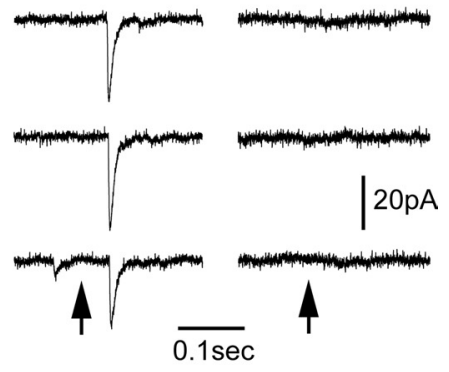

D
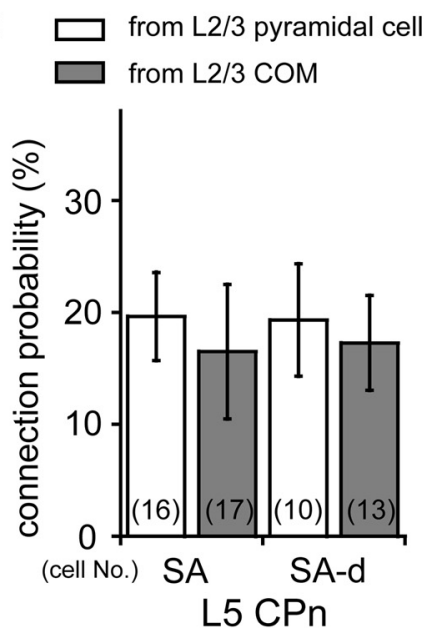

Figure 6. Interlaminar connections from $L 2 / 3$ to $L 5$ COM cells. $A$, Connections from $L 2 / 3$ to $L 5$ cells were examined using focal glutamate puff stimulation (left). Right, DIC image of layer II/III (top) and cells labeled by tracer in the same field (bottom). $\boldsymbol{B}$, EPSCS evoked at constant latency during trials by puff stimulation (left). Right, Examples of no EPSC induction by the stimulation. Arrow, Onset of glutamate puff stimulation. C, Connection probabilities from L2/3 to L5 COM firing subtypes. White bars, Random L2/3 pyramidal cell stimulation; gray bars, L2/3 COM cell stimulation. L5 COM cells preferentially received inputs from L2/3 COM cells. ${ }^{* *} p<0.01$. D, Connection probabilities from L2/3 to L5 CPn cells identified by retrograde tracer injection to ipsilateral pontine nuclei. Note that L5 (Pn cells consisted of two firing (SA and SA-d) subtypes. Data in $\boldsymbol{C}$ and $\boldsymbol{D}$ are means \pm SD. The numbers in the bar graphs indicate the number of $L 5$ cells tested.

constant latencies (Otsuka and Kawaguchi, 2008). We searched for EPSCs evoked at a constant latency in L5 COM cells after glutamate stimulation to individual L2/3 cells (Fig. 6 B). Connection probability in individual L5 cells was then calculated from the number of L2/3 cells evoking EPSCs in recorded L5 cell and the total number of stimulated L2/3 cells during the recording. To examine connection selectivity from L2/3 to L5 COM cells, we compared connection probabilities in L2/3 pyramidal cell stimulations sampled randomly under differential interference contrast (DIC) imaging with those in selective L2/3 COM cell stimulation selected by retrograde fluorescent labeling (Fig. $6 A$, right images). L2/3 pyramidal cell stimulation under DIC imaging would be applied to both labeled and unlabeled cells. The mean number of stimulated L2/3 cells tested with each L5 COM subtype in random and selective cases was $32.1 \pm 8.3$ and $31.3 \pm 5.4$ for SA cells, $32.3 \pm 7.5$ and $30.3 \pm 3.9$ for SA-d cells, and $31.9 \pm 7.9$ and $31.2 \pm 5.6$ for FA cells (range, $25-50$ ).

Connection probabilities from L2/3 pyramidal to L5 COM cells depended on L5 COM firing subtypes. Consistent with our previous study (Otsuka and Kawaguchi, 2008), L5 FA type COM cells received inputs from L2/3 pyramidal cells with a lower connection probability than those in SA and SA-d type COM cells regardless of whether stimulated L2/3 cells were randomly selected (Fig. $6 C$, open columns) $(p<0.01)$ or restricted to COM cells (Fig. $6 C$, filled columns) $(p<0.05)$. Importantly, connection probabilities from L2/3 COM to L5 COM cells were signifi- cantly higher than those from randomly stimulated L2/3 cells to L5 COM cells $(20.49 \pm 7.97$ vs $10.16 \pm 2.98$ for $\mathrm{FA}$; $25.04 \pm 6.17$ vs $17.92 \pm 3.77$ for SA; and $25.35 \pm 7.04$ vs $17.92 \pm 4.85$ for SA-d type; $p<0.01$ in all cases). These results suggest that L5 COM cells receive synaptic inputs preferentially from L2/3 COM cells.

To further address whether L2/3 COM cells preferentially innervate L5 COM cells, we examined connections from L $2 / 3$ to L5 pyramidal cells that project to the ipsilateral pontine nuclei [corticopontine (CPn) cells], which include brainstem projection neurons (Cowan and Wilson, 1994). CPn cells consist of SA and SA-d firing subtypes (Otsuka and Kawaguchi, 2008). Dual injections of retrograde fluorescent tracers to the contralateral frontal cortex and ipsilateral pontine nuclei from the recording site revealed nonoverlapped distribution of CPn and COM cells in L5 (data not shown), confirming that these two types are separate populations (Hallman et al., 1988). In contrast with interlaminar connections from L2/3 to L5 COM cells, connection probabilities from L2/3 pyramidal cells to L5 CPn cells were similar between SA and SA-d type cells in both random L2/3 pyramidal and selective L2/3 COM cell stimulations (Fig. 6D). Connection probabilities to L5 CPn cells did not depend on L2/3 stimulations to random selected pyramidal cell or COM cells $(19.65 \pm 3.94$ vs $16.5 \pm 5.99$ for SA; and $19.32 \pm 5.01$ vs $17.27 \pm 4.23 \%$ for SA-d; $p>0.05)$. We stimulated $31.2 \pm 5.0$ and $31.1 \pm 4.9 \mathrm{~L} 2 / 3$ cells for SA type cells and $32.3 \pm 7.7$ and $32.2 \pm 6.0 \mathrm{~L} 2 / 3$ cells for SA-d type cells in random and selective cases, respectively (range, 25-45 cells). These results suggest that L5 CPn cells receive synaptic inputs from L2/3 cells independently whether presynaptic cells are COM cells or not. Moreover, connection probabilities from L2/3 COM cells to SA and SA-d type L5 COM cells were significantly higher than those to SA and SA-d type L5 CPn cells, respectively $(p<0.01)$. Together, these results suggest that $\mathrm{L} 2 / 3$ COM cells preferentially innervate L5 COM cells.

\section{Interlaminar connection specificity from $\mathrm{L} 2 / 3$ to $\mathrm{L} 5$ COM cells}

Interlaminar connections from L2/3 to L5 pyramidal cells are clustered into subnetworks, depending on the connectivity and cell firing subtypes in layer 5 (Kampa et al., 2006; Otsuka and Kawaguchi, 2008). However, it remains unknown whether connection specificity from L2/3 to L5 cells depends on projection cell subtypes. To address this, we obtained dual whole-cell recordings from neighboring L5 COM cells, while glutamate stimulation was applied to L2/3 COM cells. Connection specificity was estimated from common input probabilities that two L 5 cells receive synaptic inputs from the same $\mathrm{L} 2 / 3$ cell. If $\mathrm{L} 5$ cells receive inputs from the same L2/3 cell, synchronous synaptic inputs should be observed in recording L5 cells (Otsuka and Kawaguchi, 2008, 2009). We searched for L2/3 COM cells that evoked syn- 
A

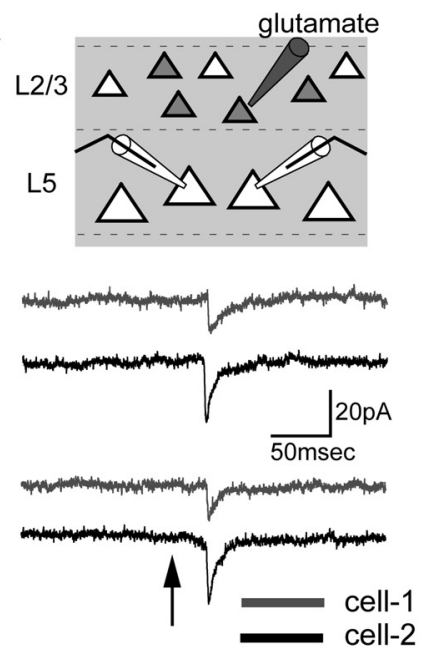

B

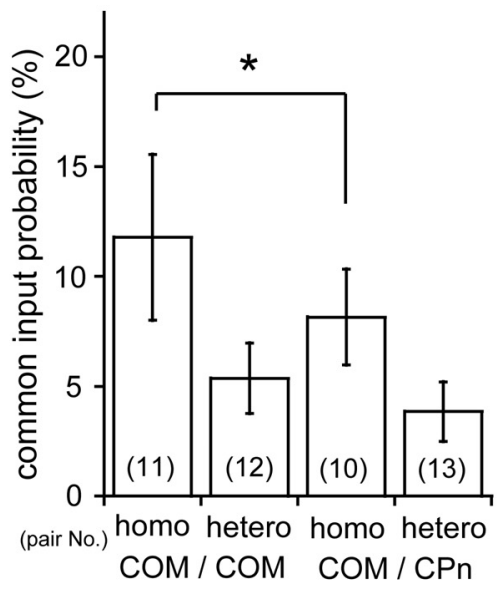

C

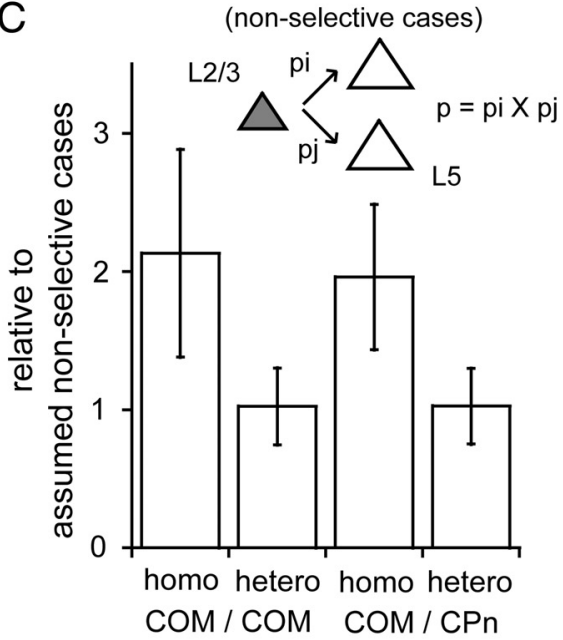

Figure 7. Interlaminar connection specificity from L2/3 to $\mathrm{L} 5$ cells. $A$, Top, Dual whole-cell recording from $\mathrm{L} 5 \mathrm{COM} / \mathrm{COM}$ or $\mathrm{COM} / \mathrm{CPn}$ cell pairs during glutamate puffstimulation to $\mathrm{L} 2 / 3 \mathrm{COM}$ cells. Bottom, Simultaneous EPSCs induced in two L5 cells by L2/3 COM stimulation. Arrow, Onset of glutamate puff stimulation. $B$, Common input probability in L5 cell pairs from L2/3 COM cells. ${ }^{*} p<$ 0.05. Homo and hetero pairs were significantly different in both $\mathrm{COM} / \mathrm{COM}$ and $\mathrm{COM} / \mathrm{CPn}(p<0.01$, not marked in the graph). The numbers in the bar graphs indicate the number of cell pairs examined. C, Common input probabilities relative to those assuming nonselective cases. Inset, Calculation of common input probabilities assuming nonselective innervations. Homo and hetero pairs were significantly different in both COM/COM and COM/CPn pairs $(p<0.01)$. Data in $B$ and $C$ are means \pm SD.

chronous inputs in recording L5 COM cells (Fig. 7A) and then calculated the common input probabilities in individual cell pairs from the number of L2/3 COM cells evoking simultaneous inputs in L 5 cells and the total number of stimulated L2/3 COM cells. To examine subtype dependency in the interlaminar connection specificity, we used common input probabilities in unconnected cell pairs. We compared common input probabilities between L5 COM cell pairs consisting of the same (homo; including $5 \mathrm{FA} / \mathrm{FA}$, $3 \mathrm{SA} / \mathrm{SA}$, and $3 \mathrm{SA}-\mathrm{d} / \mathrm{SA}-\mathrm{d}$ pairs) and different (hetero; including $5 \mathrm{FA} / \mathrm{SA}, 4 \mathrm{FA} / \mathrm{SA}-\mathrm{d}$, and $3 \mathrm{SA} / \mathrm{SA}-\mathrm{d}$ pairs) firing subtypes. The mean number of stimulated L2/3 COM cells tested in homo and hetero cell pairs was $34.9 \pm 7.0$ and $35.1 \pm 5.6$, respectively (range, 30-50 cells). Common input probabilities in L5 COM cell pairs of homo groups were significantly higher than those in hetero groups (Fig. $7 B)(11.78 \pm 3.77$ for homo group; $5.37 \pm$ $1.6 \%$ for hetero group; $p<0.01$ ), suggesting that connections from L2/3 to L5 COM cells are clustered into subnetworks, depending on the firing subtypes of L5 COM cells.

L5 COM cells consisted of heterogeneous firing subtypes that correlated with projection patterns to the contralateral and ipsilateral cortical areas. From firing subtypes and morphological properties, L5 FA type COM cells are likely to overlap with L5 pyramidal cells that project to the contralateral striatum (i.e., CCS cells), suggesting that subnetworks of L2/3 to L5 FA type COM cells correspond to the output channel to the contralateral striatum. To examine whether SA and SA-d type L5 COM cells form subnetworks with other L5 pyramidal subtypes projecting to the subcortical areas, we compared common input probabilities among cell pairs of L5 COM and CPn cells. Although CPn cells consist of SA and SA-d firing subtypes, L5 SA and SA-d type COM cells are likely to comprise different projection subtypes from CPn cells, because there is no overlap of retrogradely labeled cells from these targets (data not shown) (Hallman et al., 1988). We selectively stimulated $35.6 \pm 4.2$ and $35.1 \pm 5.7 \mathrm{~L} 2 / 3 \mathrm{COM}$ cells for homo and hetero L5 COM/CPn cell pairs (range, 30-45 cells). Common input probabilities from L2/3 COM cells to L5 $\mathrm{COM} / \mathrm{CPn}$ cell pairs of the same firing (SA/SA and SA-d/SA-d, homo pairs) subtypes were significantly higher than those of the different firing subtypes (hetero pairs) (Fig. $7 B)(8.15 \pm 2.18$ for homo pairs; $3.8 \pm 1.35 \%$ for hetero pairs; $p<0.01$ ). Common input probabilities in L5 COM/COM homo pairs were highest among pair combinations examined ( $p<0.05$, between COM/ $\mathrm{COM}$ and $\mathrm{COM} / \mathrm{CPn}$ homo pairs).

Connection probabilities from L2/3 COM to L5 COM or CPn cells differed among L5 firing subtypes (Fig. 6). To compare connection specificity between L5 cell groups, we normalized the common input probabilities for different postsynaptic cell types relative to the probabilities expected in nonselective connections from L2/3 to L5 cells (Otsuka and Kawaguchi, 2008). Common input probabilities in nonselective innervations were calculated by $p_{i} \times p_{j}$ (Fig. $7 C$, insets), where $p_{i}$ and $p_{j}$ are the experimentally determined probabilities that individual L5 cell subtypes receive inputs from L2/3 COM cells (Fig. 6). Relative common input probabilities were close to 1 in different firing subtype combinations of $\mathrm{L} 5 \mathrm{COM} / \mathrm{COM}$ and $\mathrm{COM} / \mathrm{CPn}$ cell pairs (Fig. $7 \mathrm{C}$ ) $(1.02 \pm 0.28$ for hetero COM/COM; $1.03 \pm 0.27$ for hetero $\mathrm{COM} / \mathrm{CPn} ; p=0.78$ and 0.75 , respectively, one-sample $t$ test), suggesting that L2/3 COM cells innervate these L5 hetero pairs in a nonselective manner. On the other hand, relative common input probabilities in L5 COM/COM and COM/CPn homo pairs were significantly higher than those in hetero pairs (Fig. $7 \mathrm{C}$ ) ( $2.12 \pm 0.75$ for homo COM/COM pairs; $1.96 \pm 0.53$ for homo $\mathrm{COM} / \mathrm{CPn}$ pairs; $p<0.01$, respectively). Absolute common input probabilities were significantly different between L5 COM/ $\mathrm{COM}$ and $\mathrm{COM} / \mathrm{CPn}$ homo pairs (Fig. $7 B$ ), but the relative probabilities were not (Fig. $7 C)(p>0.05)$. These results suggest that L5 SA and SA-d COM cells form interlaminar subnetworks with L5 CPn cells that do not project to the contralateral cortex.

\section{Discussion}

In this study, we investigated physiological and morphological properties of, and local connections between, COM cells in the frontal cortex to understand how the commissural system organizes the intracortical excitatory subnetworks. L2/3 COM cells were of a homogeneous population in firing patterns, but L5 $\mathrm{COM}$ cells were heterogeneous in physiological and morphological properties that correlated with projection patterns to the contralateral and ipsilateral cortical areas. We found that in- 
tralaminar connections between L5 COM cells depended on their firing subtypes: homo pairs (same firing subtype combination) were more frequently connected than were hetero pairs (different firing subtype combination). In interlaminar feedforward connections, L2/3 COM cells formed synaptic connections with L5 COM cells more frequently than with another L 5 projection subtype, CPn cells. Common input probabilities from L2/3 to L5 COM cells also depended on L5 COM subtypes. L5 COM homo cell pairs frequently received common inputs from L2/3 COM cells compared with those in hetero pairs. Moreover, L5 CPn cells, which do not send axonal projections to the contralateral cortex, formed interlaminar subnetworks with specific L5 COM subtypes. Our results suggest that COM cells form intralaminar and interlaminar subnetworks corresponding to corticocortical and subcortical projection patterns.

Previous studies in the mouse somatosensory and visual cortex reported physiological properties of L5 pyramidal cells projecting to the contralateral cortex that were identified in similar ways to our study (Hattox and Nelson, 2007; Ramos et al., 2008; Brown and Hestrin, 2009). Those articles, however, suggested that L5 COM cells are homogeneous in their physiological properties. In contrast, our recordings obtained from the rat frontal cortex showed heterogeneous populations. Anatomically, it is known that a subset of L5 COM cells send axonal projections to the contralateral striatum (Wilson, 1987). Therefore, if L5 COM cells were of a homogeneous population, they would have similar physiological and morphological properties with L5 pyramidal cells projecting to the contralateral striatum (CCS cells). However, some morphological and physiological differences between COM and CCS cells were reported in the mouse cortex (Hattox and Nelson, 2007; Brown and Hestrin, 2009). CCS cells have a slender type of apical dendrites and show fast spike frequency adaptation during current pulse injections (Morishima and Kawaguchi, 2006; Otsuka and Kawaguchi, 2008), indicating that FA type L5 COM cells obtained in the present study overlap with the CCS population. SA and SA-d type COM cells were morphologically different from FA type COM cells. Their apical tufts originated from the main apical shafts at a greater depth, similar to those of CPn cells (Morishima and Kawaguchi, 2006). However, apical tufts of SA and SA-d type L5 COM cells were poorly developed in comparison with those of CPn cells (Morishima and Kawaguchi, 2006). Moreover, COM cells showed heterogeneity in corticocortical projection patterns: projections to distant neocortical areas of the ipsilateral hemisphere were found in some COM cells, but not in others (Koralek et al., 1990; Mitchell and Macklis, 2005). These corticocortical projection patterns correlated with firing subtypes in the present study. Together, these results suggest that L5 COM cells are of heterogeneous populations with distinct intrinsic membrane properties, dendritic morphologies, and corticocortical projection patterns. This notion is supported by heterogeneous gene expressions among COM cells within individual layers (Molyneaux et al., 2009).

How intracortical circuits generate diverse outputs for multiple brain areas remains unknown. Recent anatomical studies have shown that pyramidal cells form dendritic bundles that relate to their axonal projection targets, suggesting these assemblies receive common inputs from other cells and work as cortical output units (Innocenti and Vercelli, 2010). Indeed, interlaminar connections from L2/3 to L5 pyramidal cells are specialized depending on pyramidal subtypes (Otsuka and Kawaguchi, 2008; Anderson et al., 2010). Similarly, intralaminar connections between L5 cortical pyramidal cells depend on their long-range projection targets (Morishima and Kawaguchi, 2006; Brown and
Hestrin, 2009), suggesting functionally segregated networks within cortical circuits. However, single L5 pyramidal cell projects to multiple brain areas (Lévesque and Parent, 1998; Veinante and Deschênes, 2003). Here, we demonstrated that connections between L5 COM cells depend on COM cell subtypes that correlate with their projection patterns to the contralateral and ipsilateral cortical areas. Moreover, interlaminar feedforward excitatory pathways from L2/3 to L5 COM cells were partially segregated into subnetworks, depending on L5 COM subtypes. These results suggest the existence of preciously differentiated subnetworks corresponding to diverse outputs to various cortical and subcortical targets.

Although the precise functional roles of interhemispheric cortical communications remain unclear, each cortical hemisphere can either inhibit or excite the other (Bloom and Hynd, 2005). In the case of inhibition, interhemispheric communications would act for the functional predominance in one hemisphere. In contrast, excitation from callosal inputs would facilitate information integration and cooperation between hemispheres. Moreover, in the visual cortex, interhemispheric callosal inputs modulate specific visual responses where inhibitory and excitatory effects are involved (Makarov et al., 2008; Nakamura et al., 2008; Schmidt et al., 2010). Consistent with these bidirectional effects, callosal fibers innervate both excitatory cells and inhibitory interneurons (Kawaguchi, 1992; Carr and Sesack, 1998; Karayannis et al., 2007; Petreanu et al., 2007). It would be interesting to know whether callosal fibers originated from particular subnetworks observed in this study selectively innervate specific types of cortical neurons in the contralateral cortical hemisphere.

In the frontal cortex, L5 pyramidal cells projecting to the subcortical areas have been divided into two major types, CCS and CPn cells (Cowan and Wilson, 1994; Levesque et al., 1996; Reiner et al., 2003; Morishima and Kawaguchi, 2006). Subnetworks of FA type COM/CCS cells would process the information for the contralateral striatum as well as the contralateral cortex and ipsilateral distant neocortical areas. CPn cells include pyramidal cells innervating other brainstem nuclei and spinal cord, but do not project to the contralateral hemisphere. CPn cells receive convergent inputs from diverse L2/3 pyramidal cells (Otsuka and Kawaguchi, 2008) and unidirectional excitations from L5 CCS cells (Morishima and Kawaguchi, 2006). How does the CPn interlaminar subnetwork transfer this convergent information to the other hemisphere? L5 SA and SA-d type COM cells appear to project mostly to the contralateral cortex and form interlaminar subnetworks with CPn cells. Since CPn cells cannot directly output the information to the contralateral cortex by themselves, SA and SA-d COM cells may act as relay cells for interhemispheric communication in relation to command generation to the cerebellum and brainstem/spinal cord motor systems.

\section{References}

Anderson CT, Sheets PL, Kiritani T, Shepherd GM (2010) Sublayer-specific microcircuits of corticospinal and corticostriatal neurons in motor cortex. Nat Neurosci 13:739-744.

Bloom JS, Hynd GW (2005) The role of the corpus callosum in interhemispheric transfer of information: excitation or inhibition? Neuropsychol Rev 15:59-71.

Brown SP, Hestrin S (2009) Intracortical circuits of pyramidal neurons reflect their long-range axonal targets. Nature 457:1133-1136.

Bureau I, von Saint Paul F, Svoboda K (2006) Interdigitated paralemniscal and lemniscal pathways in the mouse barrel cortex. PLoS Biol 4:e382.

Bury SD, Jones TA (2002) Unilateral sensorimotor cortex lesions in adult rats facilitate motor skill learning with the "unaffected" forelimb and training-induced dendritic structural plasticity in the motor cortex. J Neurosci 22:8597-8606. 
Carr DB, Sesack SR (1998) Callosal terminals in the rat prefrontal cortex: synaptic targets and association with GABA-immunoreactive structures. Synapse 29:193-205.

Cowan RL, Wilson CJ (1994) Spontaneous firing patterns and axonal projections of single corticostriatal neurons in the rat medial agranular cortex. J Neurophysiol 71:17-32.

DeFelipe J, Fariñas I (1992) The pyramidal neuron of the cerebral cortex: morphological and chemical characteristics of the synaptic inputs. Prog Neurobiol 39:563-607.

Gazzaniga MS (2005) Forty-five years of split-brain research and still going strong. Nat Rev Neurosci 6:653-659.

Hallman LE, Schofield BR, Lin CS (1988) Dendritic morphology and axon collaterals of corticotectal, corticopontine, and callosal neurons in layer $\mathrm{V}$ of primary visual cortex of the hooded rat. J Comp Neurol 272:149-160.

Hasegawa I, Fukushima T, Ihara T, Miyashita Y (1998) Callosal window between prefrontal cortices: cognitive interaction to retrieve long-term memory. Science 281:814-818.

Hattox AM, Nelson SB (2007) Layer V neurons in mouse cortex projecting to different targets have distinct physiological properties. J Neurophysiol 98:3330-3340.

Innocenti GM, Vercelli A (2010) Dendritic bundles, minicolumns, columns, and cortical output units. Front Neuroanat 4:11.

Kampa BM, Letzkus JJ, Stuart GJ (2006) Cortical feed-forward networks for binding different streams of sensory information. Nat Neurosci 9:1472-1473.

Karayannis T, Huerta-Ocampo I, Capogna M (2007) GABAergic and pyramidal neurons of deep cortical layers directly receive and differently integrate callosal input. Cereb Cortex 17:1213-1226.

Kawaguchi Y (1992) Receptor subtypes involved in callosally-induced postsynaptic potentials in rat frontal agranular cortex in vitro. Exp Brain Res 88:33-40.

Kawaguchi Y, Kubota Y (1997) GABAergic cell subtypes and their synaptic connections in rat frontal cortex. Cereb Cortex 7:476-486.

Koralek KA, Olavarria J, Killackey HP (1990) Areal and laminar organization of corticocortical projections in the rat somatosensory cortex. J Comp Neurol 299:133-150.

Larson EB, Burnison DS, Brown WS (2002) Callosal function in multiple sclerosis: bimanual motor coordination. Cortex 38:201-214.

Lefort S, Tomm C, Floyd Sarria JC, Petersen CC (2009) The excitatory neuronal network of the $\mathrm{C} 2$ barrel column in mouse primary somatosensory cortex. Neuron 61:301-316.

Lévesque M, Parent A (1998) Axonal arborization of corticostriatal and corticothalamic fibers arising from prelimbic cortex in the rat. Cereb Cortex 8:602-613.

Levesque M, Charara A, Gagnon S, Parent A, Deschenes M (1996) Corticostriatal projections from layer $\mathrm{V}$ cells in rat are collaterals of long-range corticofugal axons. Brain Res 709:311-315.

Lübke J, Feldmeyer D (2007) Excitatory signal flow and connectivity in a cortical column: focus on barrel cortex. Brain Struct Funct 212:3-17.

Makarov VA, Schmidt KE, Castellanos NP, Lopez-Aguado L, Innocenti GM
(2008) Stimulus-dependent interaction between the visual areas 17 and 18 of the 2 hemispheres of the ferret (Mustela putorius). Cereb Cortex 18:1951-1960.

McCormick DA, Pape HC (1990) Properties of a hyperpolarizationactivated cation current and its role in rhythmic oscillation in thalamic relay neurones. J Physiol 431:291-318.

Mitchell BD, Macklis JD (2005) Large-scale maintenance of dual projections by callosal and frontal cortical projection neurons in adult mice. J Comp Neurol 482:17-32.

Molyneaux BJ, Arlotta P, Fame RM, MacDonald JL, MacQuarrie KL, Macklis JD (2009) Novel subtype-specific genes identify distinct subpopulations of callosal projection neurons. J Neurosci 29:12343-12354.

Morishima M, Kawaguchi Y (2006) Recurrent connection patterns of corticostriatal pyramidal cells in frontal cortex. J Neurosci 26:4394-4405.

Mountcastle VB (1997) The columnar organization of the neocortex. Brain 120:701-722.

Nakamura H, Chaumon M, Klijn F, Innocenti GM (2008) Dynamic properties of the representation of the visual field midline in the visual areas 17 and 18 of the ferret (Mustela putorius). Cereb Cortex 18:1941-1950.

Otsuka T, Kawaguchi Y (2008) Firing-pattern-dependent specificity of cortical excitatory feed-forward subnetworks. J Neurosci 28:11186-11195.

Otsuka T, Kawaguchi Y (2009) Cortical inhibitory cell types differentially form intralaminar and interlaminar subnetworks with excitatory neurons. J Neurosci 29:10533-10540.

Petreanu L, Huber D, Sobczyk A, Svoboda K (2007) Channelrhodopsin-2assisted circuit mapping of long-range callosal projections. Nat Neurosci 10:663-668.

Ramos RL, Tam DM, Brumberg JC (2008) Physiology and morphology of callosal projection neurons in mouse. Neuroscience 153:654-663.

Reiner A, Jiao Y, Del Mar N, Laverghetta AV, Lei WL (2003) Differential morphology of pyramidal tract-type and intratelencephalically projecting-type corticostriatal neurons and their intrastriatal terminals in rats. J Comp Neurol 457:420-440.

Schmidt KE, Lomber SG, Innocenti GM (2010) Specificity of neuronal responses in primary visual cortex is modulated by interhemispheric corticocortical input. Cereb Cortex 20:2776-2786.

Song S, Sjöström PJ, Reigl M, Nelson S, Chklovskii DB (2005) Highly nonrandom features of synaptic connectivity in local cortical circuits. PLoS Biol 3:e68.

Veinante P, Deschênes M (2003) Single-cell study of motor cortex projections to the barrel field in rats. J Comp Neurol 464:98-103.

Weiler N, Wood L, Yu J, Solla SA, Shepherd GM (2008) Top-down laminar organization of the excitatory network in motor cortex. Nat Neurosci 11:360-366.

Wilson CJ (1987) Morphology and synaptic connections of crossed corticostriatal neurons in the rat. J Comp Neurol 263:567-580.

Yoshimura Y, Dantzker JL, Callaway EM (2005) Excitatory cortical neurons form fine-scale functional networks. Nature 433:868-873. 